\title{
A FLEXIBLE, LARGE-SCALE DIAMOND-POLYMER CHEMICAL SENSOR FOR NEUROTRANSMITTER DETECTION
}

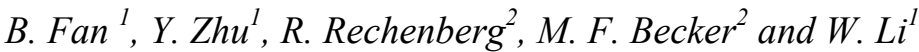 \\ ${ }^{1}$ Michigan State University, East Lansing, Michigan, USA \\ ${ }^{2}$ Fraunhofer USA, Inc - Center for Coating and Diamond Technologies, East Lansing, USA
}

\section{ABSTRACT}

This paper reports a flexible, diamond-polymer chemical sensor with three microelectrodes, which features a wide aqueous potential window and low background current from diamond and mechanical flexibility from the polymer. An improved wafer transfer process is developed to transfer boron-doped polycrystalline diamond (BDD) onto a thin Parylene-C substrate with good uniformity, strong adhesion, and high yield. The as-fabricated sensor shows a much wider potential window compared to an $\mathrm{Au}$ electrode, and a sensitivity of $\sim 0.018 \mathrm{~mA} / \mathrm{mm}^{2} \cdot \mathrm{mM}$ through cyclic voltammetry for $\mathrm{Ru}\left(\mathrm{NH}_{3}\right)_{6}{ }^{2+/ 3+}$ and $0.0012 \mu \mathrm{A} / \mathrm{mm}^{2} \cdot \mu \mathrm{M}$ through chronoamperometry for dopamine (DA).

\section{INTRODUCTION}

The central nervous system (CNS) consists of large networks of neurons that communicate with each other via electrical impulses (action potentials) along the axon and chemicals at the synapse between neuron-to-neuron. Dysregulated neurotransmitter transmission has been linked to several neurological disorders, such as Schizophrenia, Huntington's disease, Alzheimer's disease and Parkinson's disease [1], [2]. Used as a molecular diagnosis for Parkinson's Disease, dopamine (DA) is one of the most important neurotransmitters [3]. Real-time physiological monitoring of the concentration of DA will allow researchers to better understand brain diseases and evaluate the efficacy of the therapeutics toward Parkinson's disease [3].

An ideal implanted neural chemical sensor for effective sensing of DA should possess the following requirements: resistance to surface biofouling, high sensitivity, biocompatibility, a wide working potential window, mechanical flexibility, and long-term stability and reliability. Boron-doped polycrystalline diamond (BDD) has shown excellent electrochemical properties in these requirements. Unlike carbon fiber, which is composed almost exclusively of $\mathrm{sp}^{2}$ carbon, requires time-consuming surface pretreatment and suffers from surface molecular adsorption, BDD shows excellent resistance to surface fouling and needs less/no pretreatment for surface activation due to $\mathrm{sp}^{3}$ carbon [4]. Besides, $\mathrm{BDD}$ has relative small double-layer capacitances (ranging from 5 to $40 \mu \mathrm{F} / \mathrm{cm}^{2}$ ) [5], which can minimize non-Faradaic current (background noise) and achieve a higher signal-to-noise ratio. The biocompatibility has been demonstrated and is comparable with titanium and 315 stainless steel [6] by observing a very small amount of adsorbed and denatured fibrinogen on the diamond surface, which is used as a biocompatibility indicator [6]. In addition, BDD has a wide potential window range from $2.27 \mathrm{~V}$ to $3.3 \mathrm{~V}$ in an aqueous environment [7], [8] and chemical inertness [9]. However, BDD is a rigid material with a Young's modulus of $\sim 1000 \times 10^{9} \mathrm{~Pa}[10]$, which is several orders of magnitude higher than that of brain tissue (a Young's modulus ranges from $\sim 10^{3}$ to $10^{5} \mathrm{~Pa}$ [11]). Such a mechanical property mismatch causes tissue irritation and damage during long-term application, which is an obstacle for the application of biochemical sensors. Additionally, BDD cannot be synthesized onto polymer substrates directly due to its high synthesis temperatures of $500-900^{\circ} \mathrm{C}$. Considering these limitations, previously, we developed a microfabrication process to transfer
BDD onto Parylene-C substrates [12] (with a Young's modules of $2.8 \times 10^{9} \mathrm{~Pa}$ [13]), where Parylene anchors were introduced to increase the bonding strength between Parylene and BDD. The BDD was released by etching silicon (Si) using potassium hydroxide $(\mathrm{KOH})$ with a custom-made jig. The capability of powering up a $\mu$ LED was also demonstrated and intended for use in optogenetics, which uses light to manipulate electrophysiological activities of neurons. In this paper, we improved this wafer transfer process by replacing wet $\mathrm{KOH}$ etching with deep-reactive-ion dry etching (DRIE) to achieve higher yield, better uniformity and adhesion. The application of this paper is dedicated to neural chemical sensing, especially, to DA detection.

The concept diagram is shown in Figure 1. A 3-electrode BDD (in black) chemical sensor fabricated on a Parylene substrate (in gray) with a reference electrode (RE), working electrode (WE) and counter electrode $(\mathrm{CE})$, is used to demonstrate the proposed fabrication method. The openings $(30 \mu \mathrm{m} \times 50 \mu \mathrm{m}$, in white) encircled in green dash lines are created on large BDD patterns to make Parylene anchors. The overall design dimensions of the sensor are $4 \mathrm{~mm} \times 4.7 \mathrm{~mm}$, and the effective surface areas of WE, CE and RE are $0.8 \mathrm{~mm}^{2}, 0.48 \mathrm{~mm}^{2}$, and $0.48 \mathrm{~mm}^{2}$, respectively.

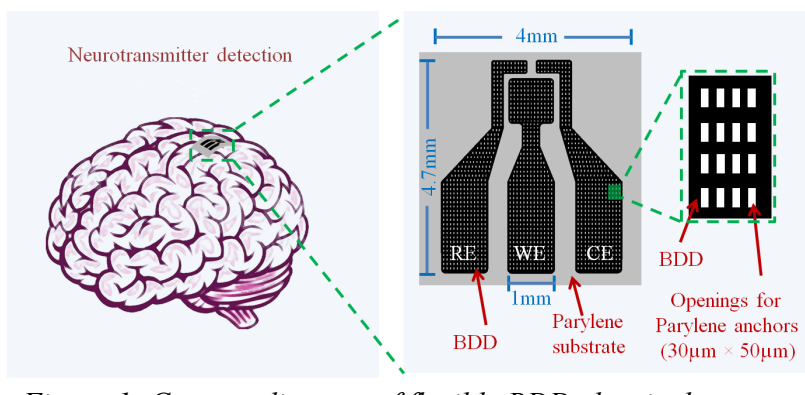

Figure 1: Concept diagram of flexible BDD chemical sensors with design dimensions.

\section{FABRICATION METHOD}

Figure 2 shows a detailed process flow. (a) One micron $(1 \mu \mathrm{m})$ sacrificial $\mathrm{SiO}_{2}$ was deposited on a 3 inch $\mathrm{Si}$ wafer using plasma enhanced chemical vapor deposition (PECVD) (PlasmaLab 80 plus ${ }^{\circledR}$, Oxford Instruments), followed by synthesis of a $\sim 2.7 \mu \mathrm{m}$ microcrystalline BDD film using a custom-made microwave plasma assisted chemical vapor deposition reactor (MPACVD) with a gas mixture of hydrogen diborane and methane. (b) A $1.3 \mu \mathrm{m}$ thick aluminum hard mask for diamond etching was deposited using a sputtering system (Denton Desk Top Pro Sputtering System, Denton Vacuum, Inc). (c) The aluminum layer was patterned using standard ultraviolet (UV) photolithography and etched in the aluminum etchant (Type A), followed by plasma etching in an electro cyclotron resonance reactive ion etcher (ECR-RIE) (Lambda Technologies, Inc.) using $\mathrm{SF}_{6} / \mathrm{Ar} / \mathrm{O}_{2}$ as processing gases, to pattern the BDD film. Then the aluminum hard mask was stripped in the aluminum etchant. (d) $\mathrm{SiO}_{2}$ was over-etched in buffered oxide etchant (BOE) to create undercuts for Parylene anchors. (e) The wafer was treated with Silane A174 adhesion promoter (Sigma Aldrich, Inc) to increase the adhesion between Parylene and BDD, 
(a)

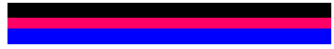

(e)

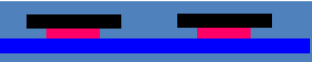

(b)

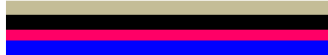

(f)

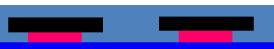

(c)

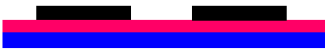

(g)

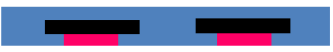

(d)

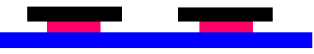

(h)

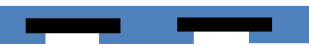

Silicon

BDD

$\mathrm{SiO}_{2}$

Parylene

\section{$\mathrm{Al}$}

Figure 2: Device fabrication and BDD transfer processes: (a) $1 \mu \mathrm{m} \mathrm{SiO}{ }_{2}$ deposition on a 3 inch silicon wafer using PECVD and $2.7 \mu \mathrm{m}$ BDD synthesis using MPACVD; (b) $1.3 \mu \mathrm{m}$ Al deposition as hard mask using DC sputtering system; (c) Al hard mask patterned using photolithography and BDD etched using ECR-RIE; (d) $\mathrm{SiO}_{2}$ undercut in BOE; (e) $17 \mu \mathrm{m}$ Parylene-C conformal coating using CVD; (f) Backside Parylene-C removal using plasma etching; (g) Silicon etching in DRIE; (h) $\mathrm{SiO}_{2}$ removal in $B O E$.

followed by a $\sim 17 \mu \mathrm{m}$ conformal Parylene-C coating (PDS 2010, Specialty Coating System, Inc). At this point, Parylene anchors were formed and wrap around BDD patterns. (f) The backside Parylene was etched in $\mathrm{O}_{2}$ plasma (PX-250 plasma system, Nordson March, Inc.). (g) The front-side of the wafer was bonded onto a carrier wafer using polyphenyl ether and the silicon substrate was completely etched from the backside using deep reactive-ion etching (DRIE). Then the BDD-Parylene layer was released from the carrier wafer with acetone soak, followed by isopropanol (IPA) and deionized water (DI water) rinse. Finally, (h) the sacrificial $\mathrm{SiO}_{2}$ was etched completely in BOE solution.

\section{DEVICE CHARACTERISTICS}

Figure 3 (a) shows an as-fabricated BDD-Parylene sensor sitting on a penny with the left branch labeled as RE, the middle branch as $\mathrm{WE}$, and the right branch as $\mathrm{CE}$. The sensor layout is symmetrical to the central line, which means RE and CE have the same size. The mesh structures correspond to the openings shown in Figure 1, which enable more Parylene anchors over large surfaces to significantly enhance the adhesion between BDD and Parylene-C. Figure 3 (b) demonstrates the mechanical flexibility of the transferred BDD-Parylene film by wrapping the device on a punch
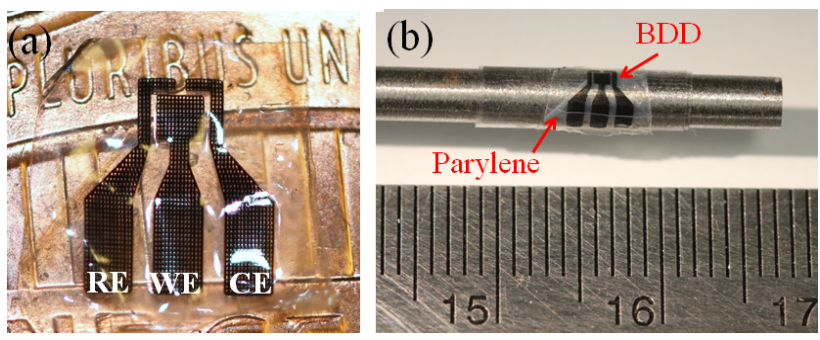

Figure 3: (a) A fabricated BDD-Parylene sensor with three electrodes. (b) A device wrapped on a $3.5 \mathrm{~mm}$-diameter punch tip, demonstrating its flexibility. tip with $3.5 \mathrm{~mm}$ in diameter.

Figure 4 (a) shows a scanning electron microscopy (SEM) image of the Parylene anchors using a low vacuum mode SEM (6610V, JEOL, Inc) with BDD in gray and Parylene in bright white. Parylene anchors which wrap around the BDD patterns are clearly observed as indicated in Figure 4 (a). The Raman spectrum taken from the nucleation side of BDD is shown in Figure 4 (b), where characteristic diamond band $\left(1332 \mathrm{~cm}^{-1}\right)$ decreases and widens and two new boron bands appear at $\sim 500 \mathrm{~cm}^{-1}$ and $\sim 1210 \mathrm{~cm}^{-1}$. The Raman features are comparable to heavily doped BDD thin films [14], where boron doping concentration is estimated at the order of $10^{20} \mathrm{~cm}^{-3}$. Energy dispersive X-ray spectrometry (EDS) was performed on the surface of BDD to confirm the complete removal of $\mathrm{SiO}_{2}$, as shown in Figure 4 (c), which otherwise causes high contact resistance and desensitizes sensing performance.
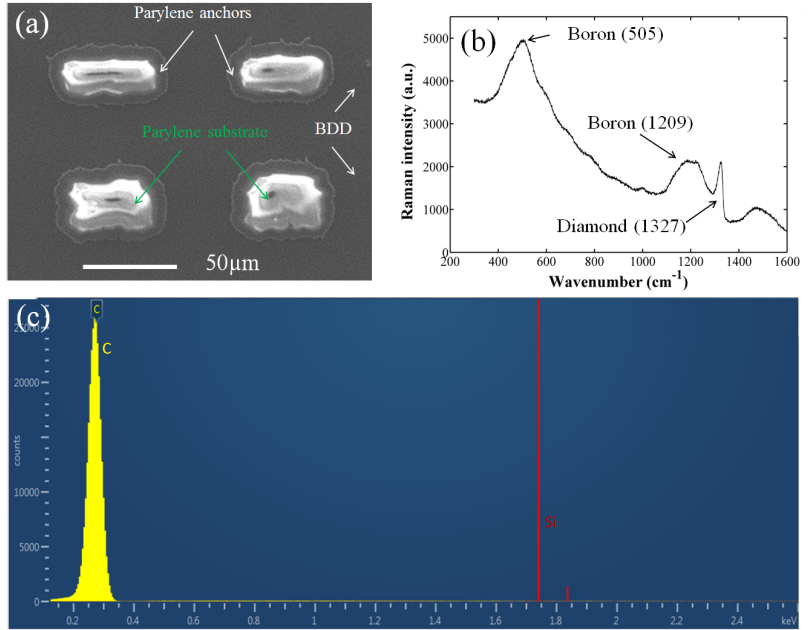

Figure 4: (a) Scanning electron microscope images show the mesh structure and Parylene anchors. (b) A Raman spectrum shows both the boron and diamond bands from the BDD nucleation side. (c) An EDS image shows only the carbon peak, implying no $\mathrm{SiO}_{2}$ residue was detected.

The strong adhesion between BDD and Parylene was verified by "Scotch tape" testing, as shown in Figure 5. In Figure 5 (a), the BDD side of the sensor was first attached to a Scotch tape and pressed to make sure the whole pattern was in good contact with the tape. Then, the BDD sensor was peeled off from the Scotch tape (Figure 5 (b)). No BDD delamination was observed after five peelings.
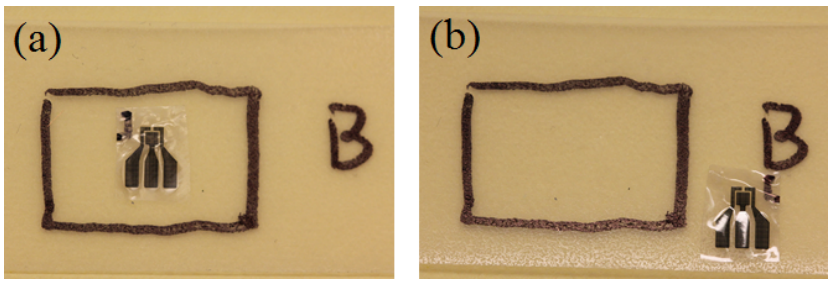

Figure 5: Scotch tape testing shows: (a) a BDD-Parylene sensor attached on to a piece of tape, with the BDD side facing down, and pressed; (b) a clean tape surface after peeling off the sensor from the tape. 

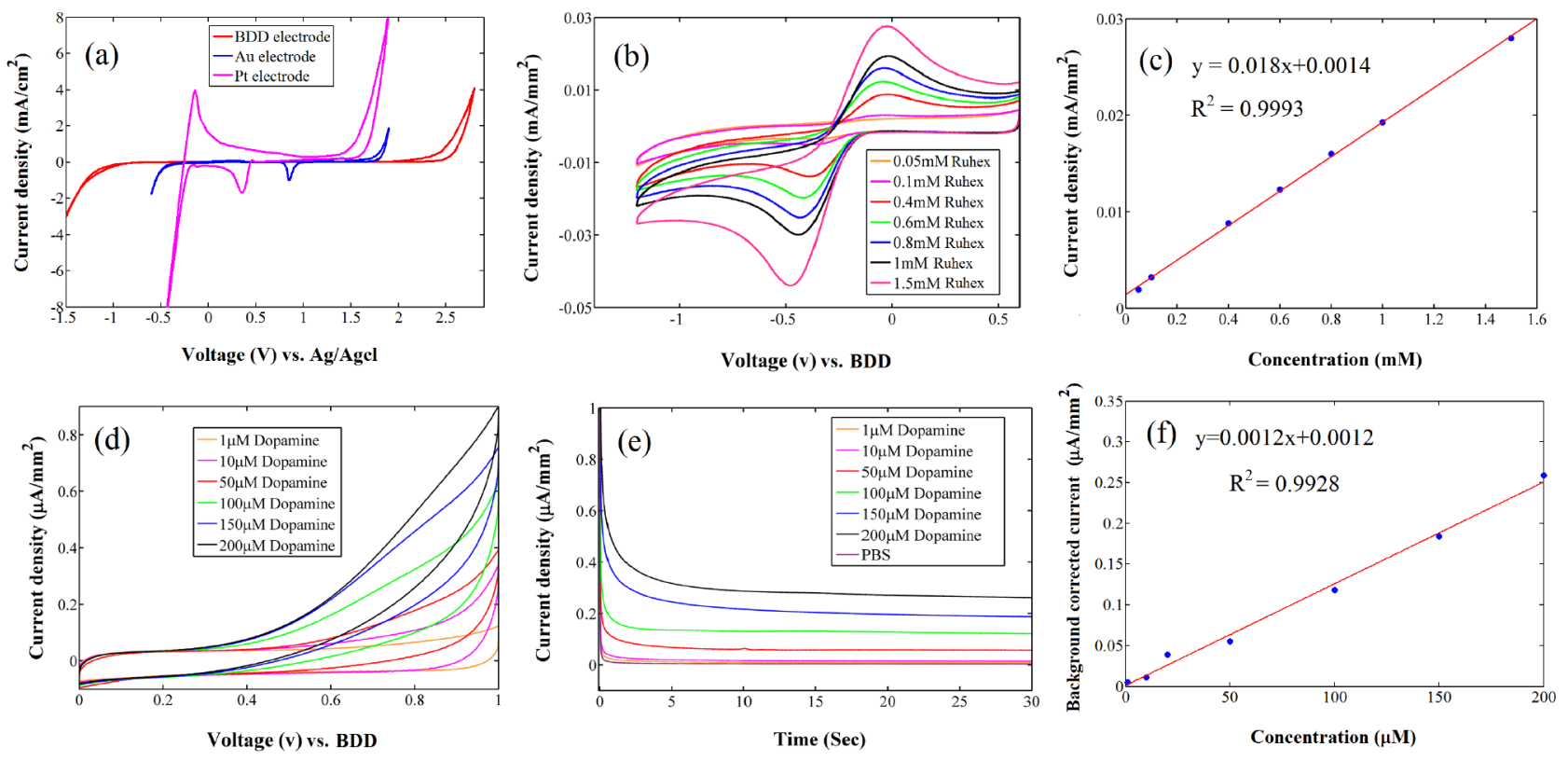

Figure 6: (a) Voltammograms of $\mathrm{Au}, \mathrm{Pt}, \mathrm{BDD}$ electrodes in 0.1 $\mathrm{M} \mathrm{H}_{2} \mathrm{SO}_{4} \mathrm{vs}$. $\mathrm{Ag} / \mathrm{AgCl}$. The scan rates are $0.1 \mathrm{~V} / \mathrm{s}$. (b) $\mathrm{CV}$ curves of the $B D D$ sensor in various concentrations of $\mathrm{Ru}\left(\mathrm{NH}_{3}\right)_{6}{ }^{2+/ 3+}$ with $1.0 \mathrm{M} \mathrm{KCl}$ solution $v$ s. BDD. The scan rate is $30 \mathrm{~V} / \mathrm{s}$. (c) Fitting curve of the cathodic peak current versus the concentrations of $\mathrm{Ru}\left(\mathrm{NH}_{3}\right)_{6}{ }^{2+/ 3+}$ (d) Voltammograms of the BDD sensor in various concentrations of $D A$ with $0.1 M$ PBS vs. BDD. The scan rates are 1.0V/s. (e) Chronoamperograms of various concentrations of DA in PBS buffer solution vs. BDD at an applied potential of 1.0V. (f) Fitting curve of the background corrected current versus the concentrations of DA.

\section{CHEMICAL SENSING TESTING}

To demostrate the low background level and wide potential window of the BDD, a standard gold electrode $(\mathrm{Au})$ and a platinum electrode $(\mathrm{Pt})$ are used as comparisons. Cyclic voltammetry $(\mathrm{CV})$ was conducted in $0.1 \mathrm{M}$ sulfuric acid $\left(\mathrm{H}_{2} \mathrm{SO}_{4}\right)$ solution vs $\mathrm{Ag} / \mathrm{AgCl}$ with a scan rate of $0.1 \mathrm{~V} / \mathrm{s}$ as shown in Figure 6 (a). The BDD electrode shows low and featureless background current from $-0.8 \mathrm{~V}$ to $2 \mathrm{~V}$. The $\mathrm{Au}$ electrode shows reduction current at $0.8 \mathrm{~V}$ and oxidation current at potentials higher than $1.4 \mathrm{~V}$. The Pt electrode shows a reduction current at $0.4 \mathrm{~V}$ and strong oxidation current at potentials higher than $1.5 \mathrm{~V}$. This demonstrates that the BDD electrode possesses lower background current and a wider potential window than the $\mathrm{Au}$ and $\mathrm{Pt}$ electrodes.

$\mathrm{Ru}\left(\mathrm{NH}_{3}\right)_{6}{ }^{2+/ 3+}$ is commonly used in electrochemistry to evaluate the reactivity of electrodes. It is classified as an outer-sphere redox couple, which is insensitive to the electrode surface microstructure, adsorption, and oxides [16]. In this case, the electron transfer rate is mainly affected by electronic properties of the electrode. Cyclic voltammetry was performed in various concentrations of $\mathrm{Ru}\left(\mathrm{NH}_{3}\right)_{6}{ }^{2+/ 3}$ in 1.0 M KCL solution using BDD with a scan rate of $30 \mathrm{~V} / \mathrm{s}$, as shown in Figure 6 (b). The well-defined oxidation and reduction peaks were observed. The peak separation is $59 \mathrm{mV}$ for reversible electron transfer systems [15]. However, our BDD sensor exhibits higher peak separation values of $\sim 400 \mathrm{mV}$, which suggests possible IR drop in the system or low electron transfer kinetics [17]. The cathodic peak currents versus various concentration of $\mathrm{Ru}\left(\mathrm{NH}_{3}\right)_{6}{ }^{2+/ 3+}$ were measured in Figure 6 (c), which implies a good linearity of the sensor response to $\mathrm{Ru}\left(\mathrm{NH}_{3}\right)_{6}{ }^{2+/ 3+}$ with an $\mathrm{R}^{2}$ value of 0.999 .

Dopamine (DA) can be detected electrochemically according to the reaction in Figure 7 , where DA is oxidized to Dopamine-o-quinone [18]. In the CV measurements (CHI 604E), the BDD WE was used to detect various concentrations of DA in
$0.1 \mathrm{M} \mathrm{pH}=7$ phosphate buffer solution (PBS) vs. BDD, with a scan rate of $1.0 \mathrm{~V} / \mathrm{s}$, as shown in Figure $6(\mathrm{~d})$. Unlike $\mathrm{Ru}\left(\mathrm{NH}_{3}\right)_{6}{ }^{2+/ 3+}$, where faster electron transfers were observed and the system was under mass transfer control by showing a peak-type voltammogram, DA detection had a relative sluggish electron transfer process with no peak observed. Chronoamperometry was conducted at a single step with a constant applied potential of $1.0 \mathrm{~V}$, and measured current decayed exponentially with time. Chronoamperograms of various concentrations of $\mathrm{DA}$ in $0.1 \mathrm{M} \mathrm{pH}=7 \mathrm{PBS}$ buffer vs. BDD is shown in Figure 6 (e). Background corrected current, which is the steady state current of chronoamperograms subtracting the current of background PBS buffer solution, versus different concentrations of DA is plotted in Figure 6 (f), where a linear response of the BDD sensor to DA was observed with an $\mathrm{R}^{2}$ value of 0.993 .

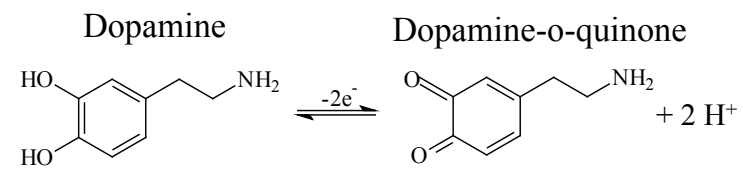

Figure 7. Dopamine / Dopamine-o-quinone redox.

\section{CONCLUSIONS}

In summary, we reported a flexible, diamond-polymer chemical sensor with three microelectrodes. An improved wafer transfer process of replacing wet $\mathrm{KOH}$ etching, as we reported previously, with deep-reactive-ion dry etching (DRIE) is used to transfer boron-doped polycrystalline diamond (BDD) onto a thin Parylene-C substrate with good uniformity, strong adhesion, and high yield. The flexibility of the as-fabricated BDD sensor was demonstrated by wrapping the sensor onto the tip of a micro punch. The Parylene anchors were observed clearly from an SEM image. 
The bonding strength was testified using a "Scotch tape" method. After five cycles of attaching, pressing and peelings, no delamination between Parylene and BDD was observed, which implies the efficacy of the Parylene anchors. Raman spectroscopy showed a typical heavily doped diamond spectrum with the diamond characteristic band at $1327 \mathrm{~cm}^{-1}$ and the two boron bands at $505 \mathrm{~cm}^{-1}$ and $1209 \mathrm{~cm}^{-1}$. The chemical performance of such a flexible BDD sensor was first reported. A wide aqueous potential window and a lower background current in the $0.1 \mathrm{M} \mathrm{H}_{2} \mathrm{SO}_{4}$ was observed as compared to a commonly used gold electrode. A faster electron transfer process under mass transfer control was observed in $\mathrm{Ru}\left(\mathrm{NH}_{3}\right)_{6}{ }^{2+/ 3+}$ redox and a sluggish electron transfer process was observed for Dopamine/Dopamine-o-quinone redox. A sensitivity of $\sim 0.018 \mathrm{~mA} / \mathrm{mm}^{2} \cdot \mathrm{mM}$ for $\mathrm{Ru}\left(\mathrm{NH}_{3}\right)_{6}{ }^{2+/ 3+}$ and $0.0012 \mu \mathrm{A} / \mathrm{mm}^{2} \cdot \mathrm{mM}$ for DA was measured experimentally.

\section{ACKNOWLEDGEMENT}

This work was supported by the National Science Foundation under the Award Numbers CBET-1264772 and ECCS-1407880. Travel support has been generously provided by the Transducer Research Foundation.

\section{REFERENCES}

[1] S. R. Ali, Y. Ma, R. R. Parajuli, Y. Balogun, W. Y.-C. Lai, and H. He, "A Nonoxidative Sensor Based on a Self-Doped Polyaniline/Carbon Nanotube Composite for Sensitive and Selective Detection of the Neurotransmitter Dopamine," Anal. Chem., vol. 79, no. 6, pp. 2583-2587, Mar. 2007.

[2] R. M. Wightman, L. J. May, and A. C. Michael, "Detection of Dopamine Dynamics in the Brain," Anal. Chem., vol. 60, no. 13, p. 769A-793A, Jul. 1988.

[3] H.-F. Cui, J.-S. Ye, Y. Chen, S.-C. Chong, and F.-S. Sheu, "Microelectrode Array Biochip: Tool for In Vitro Drug Screening Based on the Detection of a Drug Effect on Dopamine Release from PC12 Cells," Anal. Chem., vol. 78, no. 18, pp. 6347-6355, Sep. 2006.

[4] B. Duran, R. F. Brocenschi, M. France, J. J. Galligan, and G. M. Swain, "Electrochemical Activation of Diamond Microelectrodes: Implications for the In Vitro Measurement of Serotonin in the Bowel," Analyst, vol. 139, no. 12, pp. 3160-3166, May 2014.

[5] G. M. Swain and R. Ramesham, "The Electrochemical Activity of Boron-Doped Polycrystalline Diamond Thin Film Electrodes," Anal. Chem., vol. 65, no. 4, pp. 345-351, Feb. 1993.

[6] L. Tang, C. Tsai, W. W. Gerberich, L. Kruckeberg, and D. R. Kania, "Biocompatibility of Chemical-Vapour-Deposited Diamond," Biomaterials, vol. 16, no. 6, pp. 483-488, 1995.

[7] M. Hupert, A. Muck, J. Wang, J. Stotter, Z. Cvackova, S. Haymond, Y. Show, and G. M. Swain, "Conductive Diamond Thin-Films in Electrochemistry," Diam. Relat. Mater., vol. 12, no. 10-11, pp. 1940-1949, Oct. 2003.

[8] J. A. Bennett, J. Wang, Y. Show, and G. M. Swain, "Effect Of Sp2-Bonded Nondiamond Carbon Impurity on the Response of Boron-Doped Polycrystalline Diamond Thin-Film Electrodes," J. Electrochem. Soc., vol. 151, no. 9, pp. E306-E313, Sep. 2004.

[9] J. Xu, M. C. Granger, Q. Chen, J. W. Strojek, T. E. Lister, and G. M. Swain, "Peer Reviewed: Boron-Doped Diamond Thin-Film Electrodes," Anal. Chem., vol. 69, no. 19, p. 591A-597A, Oct. 1997.

[10] Diamond Materials, "The CVD diamond booklet." [Online]. Available:

http://www.diamond-materials.com/downloads/cvd_diamon d_booklet.pdf.
[11] P. Schiavone, F. Chassat, T. Boudou, E. Promayon, F. Valdivia, and Y. Payan, "In Vivo Measurement of Human Brain Elasticity using a Light Aspiration Device," Med. Image Anal., vol. 13, no. 4, pp. 673-678, Aug. 2009.

[12] B. Fan, R. Rechenberg, M. F. Becker, and W. Li, "Fabrication of Polycrystalline Diamond on a Flexible Parylene Substrate," in 2015 Transducers - 2015 18th International Conference on Solid-State Sensors, Actuators and Microsystems (TRANSDUCERS), 2015, pp. 892-895.

[13] "Parylene Specifications." [Online]. Available: http://vsiparylene.com/parylene-advantages/properties/.

[14] M. Bernard, A. Deneuville, and P. Muret, "Non-Destructive Determination of the Boron Concentration of Heavily Doped Metallic Diamond Thin Films from Raman Spectroscopy," Diam. Relat. Mater., vol. 13, no. 2, pp. 282-286, Feb. 2004.

[15] Allen J. Bard and Larry R. Faulkner, "Electrochemical Methods Fundamentals and Applications." Wiley; 2 edition, December 18, 2000.

[16] R. L. McCreery, "Advanced Carbon Electrode Materials for Molecular Electrochemistry," Chem. Rev., vol. 108, no. 7, pp. 2646-2687, Jul. 2008.

[17] J. Anderson, "The Temperature Dependence of the Formal Potential and the Rate Constant of The Heterogeneous Electron-Transfer Reaction of Myoglobin," Virginia Commonwealth University Richmond.

[18] B. J. Venton and R. M. M. Wightman, "Psychoanalytical Electrochemistry," Anal. Chem., vol. 75, no. 19, Oct. 2003.

\section{CONTACT}

* Wen Li, tel: +1-517-353-7832; wenli@msu.edu

* Bin Fan, tel: +1-517-355-3299; fanbin@msu.edu 